MITSUBISHI ELECTRIC RESEARCH LABORATORIES

http://www.merl.com

\title{
Combining Orthogonal Space Time Block Codes with Adaptive Sub-Group Antenna Encoding
}

\author{
Jingxian Wu, Henry Horng, Jinyun Zhang, Chengshan Xiao, Jan C. Olivier
}

TR2006-013 April 2006

\begin{abstract}
An adaptive space time transmit diversity scheme with simple feedback is proposed for the next generation wireless communication systems. By combining orthogonal space time block codes with adaptive sub-group antenna encoding, this new diversity scheme can effectively exploit the diversity potential provided by multiple antenna arrays without introducing interference among the signals transmitted at different antennas. In order to reduce the amount of feedback information as well as the computational complexity, a new quadrant phase constraining method is introduced to formulate the feedback information. With simple operations at both the transmitter and the receiver, the new adaptive diversity scheme out-performs not only open loop space time block encoding techniques, but also some close loop transmit diversity techniques with the same amount of feedback.
\end{abstract}

IJWIN 2005

This work may not be copied or reproduced in whole or in part for any commercial purpose. Permission to copy in whole or in part without payment of fee is granted for nonprofit educational and research purposes provided that all such whole or partial copies include the following: a notice that such copying is by permission of Mitsubishi Electric Research Laboratories, Inc.; an acknowledgment of the authors and individual contributions to the work; and all applicable portions of the copyright notice. Copying, reproduction, or republishing for any other purpose shall require a license with payment of fee to Mitsubishi Electric Research Laboratories, Inc. All rights reserved.

Copyright (C) Mitsubishi Electric Research Laboratories, Inc., 2006

201 Broadway, Cambridge, Massachusetts 02139 



\title{
Combining Orthogonal Space Time Block Codes with Adaptive Sub-group Antenna Encoding
}

\author{
Jingxian Wu, ${ }^{1}$ Henry Horng, ${ }^{2}$ Jinyun Zhang, ${ }^{2}$ Chengshan Xiao, ${ }^{3,5}$ and Jan C. Olivier ${ }^{4}$
}

\begin{abstract}
An adaptive space time transmit diversity scheme with simple feedback is proposed for the next generation wireless communication systems. By combining orthogonal space time block codes with adaptive sub-group antenna encoding, this new diversity scheme can effectively exploit the diversity potential provided by multiple antenna arrays without introducing interference among the signals transmitted at different antennas. In order to reduce the amount of feedback information as well as the computational complexity, a new quadrant phase constraining method is introduced to formulate the feedback information. With simple operations at both the transmitter and the receiver, the new adaptive diversity scheme outperforms not only open loop space time block encoding techniques, but also some close loop transmit diversity techniques with the same amount of feedback.
\end{abstract}

KEY WORDS: Adaptive antenna; orthogonal space time block codes; transmit diversity

\section{INTRODUCTION}

Transmit diversity is one of the key techniques adopted by high speed downlink packet access (HSDPA) [1] of the third generation partnership project (3GPP) standard to provide high speed reliable communication. One of the most commonly used transmit diversity techniques is the orthogonal space time block code (STBC) [2]. It is pointed out in [3] that for complex constellations, rate one STBC only exists for systems with exactly two transmit antennas.

Recently, a lot of efforts have been put into extending the STBC encoding scheme to systems with more than 2 transmit antennas without sacrificing the

\footnotetext{
${ }^{1}$ Department of Engineering Science, Sonoma State University, California, CA 94928, USA.

2 Mitsubishi Electric Research Lab, Cambridge, MA 02139, USA.

3 Department of ECE, University of Missouri, Columbia, MO 65211, USA.

${ }^{4}$ Department of EEE, University of Pretoria, Pretoria, South Africa.

5 Tel.: + 1-573-884-5367; Fax: +1-573-882-0397; E-mail: xiaoc@ missouri.edu
}

coding rate [4-8]. In addition, the channel state information (CSI) is utilized by some transmit diversity systems to further improve the system performance. The CSI can be made available to the transmitter through a separate feedback channel, and such encoding schemes are called close loop techniques [9-15]. Most of the existing close loop techniques require considerable amount of information transmitted in the feedback channel, e.g., vectors with complex-valued or real-valued elements, thus a lot of bandwidth of the reverse channel will be consumed by the feedback information.

In this paper, a new adaptive space time encoding scheme is proposed for systems with more than two transmit antennas, and only a very small amount of feedback information (at the order of several bits) are required by this close loop technique. To utilize the orthogonality of the STBC encoder, all of the transmit antennas are divided into two sub-groups, with each sub-group corresponding to one of the two output streams of the STBC encoder. Adaptive space encoding vectors are employed by each of the antenna sub-group to improve the overall diversity gain as well as to keep the orthogonality between the 
signals transmitted by different antenna groups. In order to reduce the amount of feedback as well as the computational complexity, a new quadrant phase constraining method is introduced to formulate the feedback information. The amounts of feedback information can be as few as 1 bit and 2 bits for systems with 3 or 4 transmit antennas, respectively, which is of practical interests for the design of next generation wireless communication systems.

The rest of this paper is organized as follows. Section 2 presents the system structure of the proposed adaptive space-time transmit diversity scheme. In Section 3, a new adaptive quadrant phase constraining method is presented for the computation of the feedback information at the receiver. Simulation results are given in Section 4, and Section 5 concludes the paper.

\section{SYSTEM STRUCTURE}

We consider a system with $M$ transmit antennas and one receive antenna. The block diagram of the baseband representation of the system is depicted in Figure 1, and only the modules directly related to the transmit diversity scheme are shown in the figure.

At the transmitter, the binary information data are first encoded and modulated, then the modulated symbols are fed into the orthogonal STBC encoder. Without loss of generality, we assume that at two consecutive symbol intervals $t_{1}$ and $t_{2}$, the input of the STBC encoder is $s_{1}$ and $s_{2}$, respectively, where $s_{j} \in \mathbb{S}$, for $j=1$, 2, with $\mathbb{S}$ being the modulation symbol set, and the energy of the modulation symbol is $E\left(\left|s_{j}\right|^{2}\right)=E_{s}$. At the STBC encoder, the input data symbols $s_{1}$ and $s_{2}$ are demultiplexed into two data streams, and the output of the STBC encoder can be written by

$$
\begin{aligned}
& \mathbf{d}_{1}=\left[\begin{array}{ll}
d_{11} & d_{12}
\end{array}\right]^{T}=\left[\begin{array}{ll}
s_{1} & s_{2}^{*}
\end{array}\right]^{T} \in \mathbb{C}^{2 \times 1}, \\
& \mathbf{d}_{2}=\left[\begin{array}{ll}
d_{21} & d_{22}
\end{array}\right]^{T}=\left[\begin{array}{ll}
s_{2} & -s_{1}^{*}
\end{array}\right]^{T} \in \mathbb{C}^{2 \times 1},
\end{aligned}
$$

where $\mathbf{d}_{k}$ corresponds to the $k$ th output stream of the STBC encoder, with $d_{k j}$ being transmitted at the time instant $t_{j},(\cdot)^{*}$ is the complex conjugate operation, and $(\cdot)^{T}$ denotes matrix transpose.

The $M$ transmit antennas are divided into two antenna sub-groups, with each sub-group corresponding to one of the output streams $\mathbf{d}_{1}$ and $\mathbf{d}_{2}$ of the STBC encoder. We assume that the number of antennas contained in the $k$ th group is $M_{k}$, for $k=1$, 2 , with $M_{1}+M_{2}=M$. Adaptive linear space encoders are employed by each of the two antenna subgroups, and they are used to map the two data streams onto the $M$ transmit antennas with the help of the channel feedback information. If we define the space encoding vector of the $k$ th sub-group as

$$
\mathbf{w}_{k}=\left[\begin{array}{llll}
w_{k, 1} & w_{k, 2} & \cdots & w_{k, M_{k}}
\end{array}\right] \in \mathbb{C}^{1 \times M_{k}}, \text { for } k=1,2,
$$

with the power constraint $\mathbf{w}_{1} \mathbf{w}_{1}^{H}+\mathbf{w}_{2} \mathbf{w}_{2}^{H}=1$, then the encoded signals to be transmitted by the

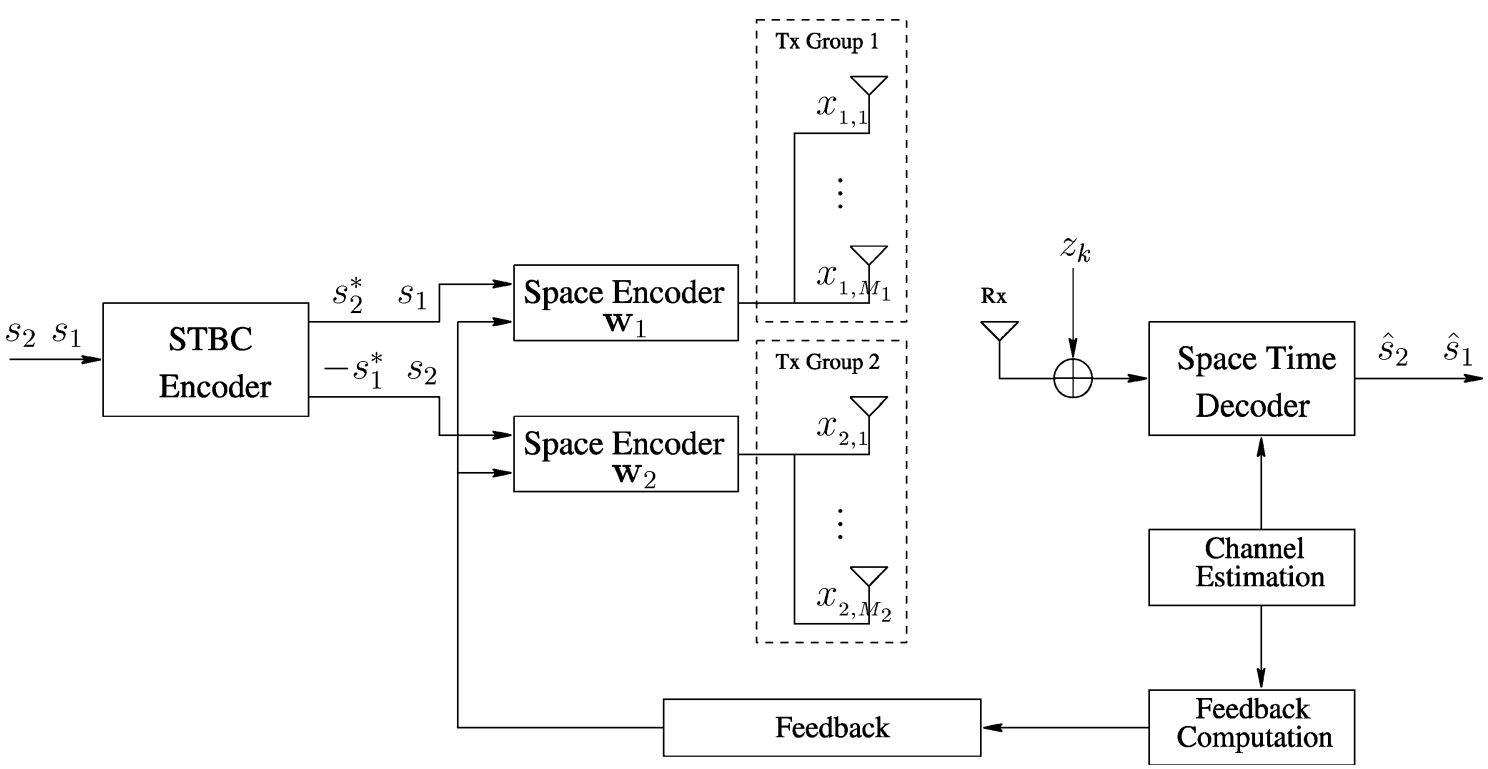

Fig. 1. The block diagram of the system with transmit diversity. 
$k$ th antenna sub-group can be written in matrix format

$$
\mathbf{X}_{k}=\mathbf{d}_{k} \cdot \mathbf{w}_{k} \in \mathbb{C}^{2 \times M_{k}}, \quad \text { for } k=1,2,
$$

with the symbols on the first row of $\mathbf{X}_{k}$ transmitted at the symbol period $t_{1}$ and symbols on the second row transmitted at $t_{2}$.

In the channel, the transmitted signals are corrupted by both the time-varying multipath fading and additive white Gaussian noise (AWGN). The signals received by the receiver are the superposition of the signals from all the transmit antennas plus noise, and they can be represented by

$$
\begin{aligned}
\mathbf{r} & =\left[\begin{array}{ll}
\mathbf{X}_{1} & \mathbf{X}_{2}
\end{array}\right]\left[\begin{array}{l}
\mathbf{h}_{1} \\
\mathbf{h}_{2}
\end{array}\right]+\mathbf{z}, \\
& =\mathbf{d}_{1} \mathbf{w}_{1} \mathbf{h}_{1}+\mathbf{d}_{2} \mathbf{w}_{2} \mathbf{h}_{2}+\mathbf{z},
\end{aligned}
$$

where $\mathbf{r}=\left[r_{1}, r_{2}\right]^{T}, \mathbf{z}=\left[z_{1}, z_{2}\right]^{T}$ are the receive vector and AWGN noise vector, respectively, with $r_{k}$ and $z_{k}$ corresponding to the time instant $t_{k}, \mathbf{h}_{k} \in \mathbb{C}^{M_{k} \times 1}$ is the channel impulse response (CIR) vector defined as

$$
\mathbf{h}_{k}=\left[\begin{array}{llll}
h_{k, 1} & h_{k, 2} & \cdots & h_{k, M_{k}}
\end{array}\right]^{T}, \quad \text { for } k=1,2,
$$

with the element $h_{k, m}$, for $m=1,2, \cdots, M_{k}$, being the CIR between the $m$ th transmit antenna of sub-group $k$ and the receive antenna.

Combining (1) and (4), we can rewrite the inputoutput relationship of the diversity system as

$$
\begin{aligned}
{\left[\begin{array}{c}
r_{1} \\
r_{2}^{*}
\end{array}\right] } & =\left[\begin{array}{cc}
\mathbf{w}_{1} \mathbf{h}_{1} & \mathbf{w}_{2} \mathbf{h}_{2} \\
-\mathbf{h}_{2}^{H} \mathbf{w}_{2}^{H} & \mathbf{h}_{1}^{H} \mathbf{w}_{1}^{H}
\end{array}\right]\left[\begin{array}{l}
s_{1} \\
s_{2}
\end{array}\right]+\left[\begin{array}{c}
z_{1} \\
z_{2}^{*}
\end{array}\right], \\
& =\mathbf{H} \cdot \mathbf{s}+\tilde{\mathbf{z}},
\end{aligned}
$$

where $\mathbf{s}=\left[s_{1} s_{2}\right]^{T}, \mathbf{z}=\left[z_{1} z_{2}^{*}\right]^{T}$ are the signal vector and noise vector, respectively, and the channel matrix $\mathbf{H}$ is defined as

$$
\mathbf{H}=\left[\begin{array}{cc}
\mathbf{w}_{1} \mathbf{h}_{1} & \mathbf{w}_{2} \mathbf{h}_{2} \\
-\mathbf{h}_{2}^{H} \mathbf{w}_{2}^{H} & \mathbf{h}_{1}^{H} \mathbf{w}_{1}^{H}
\end{array}\right] \in \mathbb{C}^{2 \times 2} .
$$

The matrix $\mathbf{H}$ is a $2 \times 2$ orthogonal matrix, i.e., $\mathbf{H}^{H} \mathbf{H}=\left(\left|\mathbf{h}_{1} \mathbf{w}_{1}\right|^{2}+\left|\mathbf{h}_{2} \mathbf{w}_{2}\right|^{2}\right) \cdot \mathbf{I}_{2}$, with $\mathbf{I}_{2}$ being a $2 \times 2$ identity matrix. From (6) and (7), we can compute the decision vector $\mathbf{y}=\left[y_{1}, y_{2}\right]^{T}$ as

$$
\begin{aligned}
\mathbf{y} & =\mathbf{H}^{H} \mathbf{r}, \\
& =\left(\left|\mathbf{w}_{1} \mathbf{h}_{1}\right|^{2}+\left|\mathbf{w}_{2} \mathbf{h}_{2}\right|^{2}\right) \cdot \mathbf{s}+\mathbf{v},
\end{aligned}
$$

where $\mathbf{v}=\mathbf{H}^{H} \mathbf{z}$ is the noise component with covariance matrix $\left(\left|\mathbf{h}_{1} \mathbf{w}_{1}\right|^{2}+\left|\mathbf{h}_{2} \mathbf{w}_{2}\right|^{2}\right) \cdot \mathbf{I}_{2} \cdot N_{0}$, and $N_{0}=E\left(\left|z_{k}\right|^{2}\right)$. With the decision variable given in (8), we can compute the signal to noise ratio (SNR) at the receiver as follows

$$
\gamma_{M}=\left(\left|\mathbf{w}_{1} \mathbf{h}_{1}\right|^{2}+\left|\mathbf{w}_{2} \mathbf{h}_{2}\right|^{2}\right) \cdot \gamma_{0},
$$

where $\gamma_{0}=\frac{E_{s}}{N_{0}}$ is the SNR without diversity. It can be seen from (9) that the SNR $\gamma$ is a function of the space encoding vectors $\mathbf{w}_{1}, \mathbf{w}_{2}$ and the CIR vectors $\mathbf{h}_{1}$, $\mathbf{h}_{2}$. By choosing appropriate forms of $\mathbf{w}_{k}$ based on the properties of the fading channels, we can improve the receiver SNR with only a small amount of feedback.

\section{ADAPTIVE SPACE ENCODING VECTOR DESIGN: QUADRANT PHASE CONSTRAINING}

\subsection{General Case}

To achieve the maximum SNR at the receiver, the optimum design criterion for the space encoding vectors $\mathbf{w}_{1}$ and $\mathbf{w}_{2}$ is

$$
\left(\mathrm{w}_{1}, \mathrm{w}_{2}\right)=\underset{\left(\mathbf{w}_{1}, \mathbf{w}_{2}\right) \in \mathcal{W}}{\operatorname{argmax}}\left\{\left|\mathbf{w}_{1} \mathbf{h}_{1}\right|^{2}+\left|\mathbf{w}_{2} \mathbf{h}_{2}\right|^{2}\right\},
$$

where $\mathcal{W}$ is the set of all the possible encoding vector pairs satisfying the power constraint $\mathbf{w}_{1} \mathbf{w}_{1}^{H}+\mathbf{w}_{2} \mathbf{w}_{2}^{H}$ $=1$. The optimum values of $\mathbf{w}_{1}$ and $\mathbf{w}_{2}$ can be obtained by exhaustively searching all the elements of the set $\mathcal{W}$. The size of the set $\mathcal{W}$ increases exponentially with the number of transmit antennas, therefore this optimum space encoding vector design method are not appropriate for systems with large number of transmit antennas.

In order to reduce the computational complexity as well as to reduce the amount of feedback information, we introduce a quadrant phase constraining method for the computation of the feedback information and the formulation of the adaptive space encoding vectors. For a general system with $M$ transmit antennas, we let $M_{1}=M_{2}=\frac{M}{2}$ if $M$ is an even number, and $M_{1}=\frac{M+1}{2}, M_{2}=\frac{M-1}{2}$ if $M$ is an odd number. Define the space encoding vector $\mathbf{w}_{k}$ as

$$
\mathbf{w}_{k}=\frac{1}{\sqrt{M}}\left[1 \exp \left(-\mathbf{i} \frac{q_{k, 2} \pi}{2}\right) \cdots \exp \left(-\mathbf{i} \frac{q_{k, M_{k}} \pi}{2}\right)\right],
$$

where $\mathbf{i}^{2}=-1$ is the imaginary part symbol, $q_{k, m} \in\{0$, $1,2,3\}$ is the feedback information, and each $q_{k, m}$ contains 2 bits of information. For systems with $M$ transmit antennas, the total number of feedback bits required by the proposed algorithm is $2 M-4$. For convenience of representation, we let $q_{1,1}=q_{2,1}=0$.

With the space encoding vectors defined in (11), we can write the corresponding SNR $\gamma_{M}$ given in (9) as 


$$
\begin{aligned}
\gamma_{M} & =\frac{1}{M} \sum_{k=1}^{2}\left|\sum_{m=1}^{M_{k}} h_{k, m} \exp \left(-\mathbf{i} \cdot q_{k, m} \frac{\pi}{2}\right)\right|^{2} \cdot \gamma_{0}, \\
& =\left(g_{c}+g_{b}\right) \cdot \gamma_{0},
\end{aligned}
$$

where the diversity gains $g_{c}$ and $g_{b}$ are given by

$$
\begin{gathered}
g_{c}=\frac{1}{M} \sum_{m=1}^{M_{1}}\left|h_{1, m}\right|^{2}+\frac{1}{M} \sum_{m=1}^{M_{2}}\left|h_{2, m}\right|^{2}, \\
g_{b}=\frac{1}{M} \sum_{m=1}^{M_{1}} \sum_{n=m+1}^{M_{1}} \Re\left[h_{1, m} h_{1, n}^{*} \exp \left(-\mathbf{i} \frac{q_{1, m}-q_{1, n}}{2} \pi\right)\right]+ \\
\frac{1}{M} \sum_{m=1}^{M_{2}} \sum_{n=m+1}^{M_{2}} \Re\left[h_{2, m} h_{2, n}^{*} \exp \left(-\mathbf{i} \frac{q_{2, m}-q_{2, n}}{2} \pi\right)\right],
\end{gathered}
$$

with $\Re(\cdot)$ being the real part operator. In the equations above, $g_{c}$ is the conventional diversity gain, which is fixed for a certain number of transmit antennas; while the feedback diversity gain $g_{b}$ varies with the value of the space encoding vector $\mathbf{w}_{k}$, which is in turn determined by the feedback information $q_{k, m}$. The value of $q_{k, m}$ can be chosen based on certain design criteria to improve the value of $g_{b}$, thus to improve the receiver SNR $\gamma_{M}$.

It can be seen from (13b) that the feedback diversity gain $g_{b}$ is expressed in a summation form. For different feedback information $q_{k, m}$, each of the summed elements in (13b) could be either positive or negative, resulting in performance enhancement or degradation, accordingly. If all the summed elements of the feedback diversity gain are non-negative, then the feedback diversity gain is guaranteed to be nonnegative. By this means, a performance gain is achieved over conventional open loop techniques.

Taking into account both the system performance and computational complexity, we are choosing a suboptimum design criterion: all of the summed elements of the feedback diversity gain $g_{b}$ are non-negative.

One of the summed elements of $g_{b}$ can be expressed as

$$
\begin{aligned}
& \Re\left[h_{k, m} h_{k, n}^{*} \exp \left(-\mathbf{i} \frac{q_{k, m}-q_{k, n}}{2} \pi\right)\right] \\
& =\left|h_{k, m}\right|\left|h_{k, n}\right| \cos \left(\Delta \theta_{k, m n}\right),
\end{aligned}
$$

where $\Delta \theta_{k, m n}=\theta_{k, m}-\theta_{k, n}-\frac{q_{k, m}-q_{k, n}}{2} \pi$, with $\theta_{k, m} \in$ $[0,2 \pi)$ being the phase of $h_{k, m}$. The term described in (14) will be non-negative if the following condition is satisfied

$$
\left|\Delta \theta_{k, m n}\right| \leq \frac{\pi}{2}, \forall m \neq n,
$$

which means the differences between all the possible angle pairs within each sub-group should be less than $\pi / 2$. One direct way to satisfy this condition is choosing the value of $q_{k, m}$ such that all the rotated phases $\theta_{k, m}-q_{k, m} \frac{\pi}{2}$, for $m=1,2, \cdots, M_{k}$, belonging to one sub-group are in the same $\pi / 2$ angle sector in a two dimensional coordinate system.

Without loss of generality, we normalize the phases of all the CIRs of the $k$ th sub-group with the phase of $\theta_{k}, 1$, and the normalized phases are represented as

$$
\tilde{\theta}_{k, m}=\theta_{k, m}-\theta_{k, 1}+2 l \pi,
$$

where the integer $l$ is chosen such that $\tilde{\theta}_{k, m}$ is in the range of $[0,2 \pi)$. The normalized phase $\tilde{\theta}_{k, m}$ is rotated clockwise by the angle of $q_{k, m} \frac{\pi}{2}$, such that the rotated angle $\tilde{\theta}_{k, m}-q_{k, m} \frac{\pi}{2}$ is in the quadrant phase sector from $[-\pi / 4, \pi / 4]$ of the coordinate system as shown in Figure 2. Following the discussions above, we can compute the feedback information $q_{k, m}$ as

$$
q_{k, m}= \begin{cases}\left\lfloor\frac{\tilde{\theta}_{k, m}+\pi / 4}{\pi / 2}\right\rfloor, & \tilde{\theta}_{k, m} \in\left[\frac{\pi}{4}, \frac{7 \pi}{4}\right), \\ 0, & \text { otherwise, }\end{cases}
$$

where $\lfloor\cdot\rfloor$ returns the nearest smaller integer. An example is given in Figure 2, where $\tilde{\theta}_{k, m}=9 \pi / 8$. From (17) we can get that $q_{k, m}=2$, and the corresponding rotated angle is $\tilde{\theta}_{k, m}-q_{k, m} \frac{\pi}{2}=\pi / 8$, which is in the angle sector of $\left[-\frac{\pi}{4}, \frac{\pi}{4}\right]$ of the coordinate system. By performing the same operations to all the normalized phases, the rotated phases of the same sub-group will be in the same quadrant angle sector, and the nonnegativity of each summed element of the diversity gain $g_{b}$ can be guaranteed. This method achieves the feedback diversity gain by constraining all the rotated phases of the CIRs of one sub-group in an angle sector of $\pi / 2$, hence we call it quadrant phase constraining method. Since the value of $q_{k, m}$ is computed separately for each of the transmit antennas, the computational complexity of this algorithm increases linearly with the number of transmit antennas.

With $q_{k, m}$ computed from (17), all the summed elements given in (14) are guaranteed to be nonnegative, and the feedback diversity gain of (13b) can be written by

$$
\tilde{g}_{b}=\frac{1}{M} \sum_{k=1}^{2} \sum_{m=1}^{M_{k}} \sum_{n=m+1}^{M_{k}}\left|h_{k, m}\right|\left|h_{k, n}^{*}\right|\left|\cos \left(\Delta \theta_{k, m n}\right)\right|,
$$




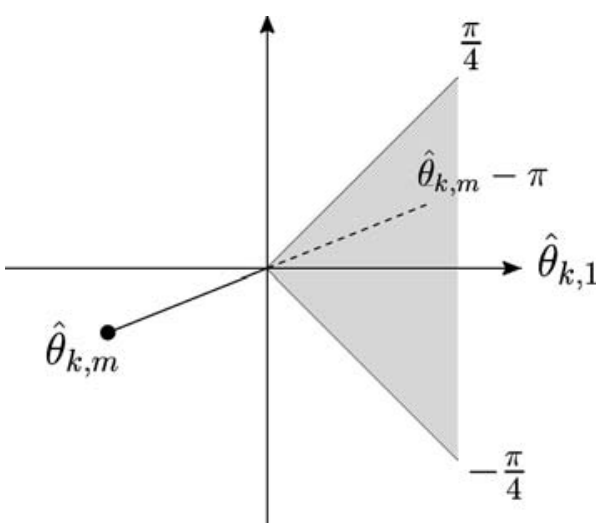

Fig. 2. The two dimensional coordinate system.

and the SNR at the receiver of the proposed algorithm is

$$
\gamma_{M}=\left(g_{c}+\tilde{g}_{b}\right) \cdot \gamma_{0},
$$

with $g_{c}$ and $\tilde{g}_{b}$ defined in (13a) and (18), respectively.

With the adaptive diversity algorithm presented in this subsection, $2 M-4$ bits of feedback information are required to form the space encoding vectors for systems with $M$ transmit antennas. It will be shown next that the amount of feedback information can be further reduced for systems with $M \leq 4$ transmit antennas, which are of practical interests of next generation communication systems.

\subsection{Special Cases: Systems with $M \leq 4$ Transmit Antennas}

For systems with $M \leq 4$ transmit antennas, each of the two antenna sub-groups will contain at most 2 transmit antennas. For sub-groups with 2 transmit antennas, the sub-optimum design criterion can be satisfied with only 1 bit of feedback information.

For systems with $M=4$ transmit antennas, the number of antennas in each of the antenna groups is $M_{1}=M_{2}=2$. We define the space encoding vector as

$$
\mathbf{w}_{k}=\frac{1}{2}\left[1(-1)^{b_{k}}\right], \quad \text { for } k=1,2,
$$

where $b_{k} \in\{0,1\}$ is the feedback information for the $k$ th antenna sub-group, and it is defined as

$$
b_{k}= \begin{cases}0, & \Re\left(h_{k, 1} h_{k_{2}}^{*}\right) \geq 0, \\ 1, & \text { otherwise }\end{cases}
$$

Combining (9), (20) and (21), we have the SNR at the receiver written by

$$
\gamma_{4}=\left(g_{4, c}+g_{4, b}\right) \gamma_{0},
$$

where the conventional diversity gain $g_{4, c}$ and the feedback diversity gain $g_{4, b}$ are defined as

$$
\begin{gathered}
g_{4, c}=\frac{1}{4}\left(\sum_{m=1}^{2}\left|h_{1, m}\right|^{2}+\sum_{m=1}^{2}\left|h_{2, m}\right|^{2}\right), \\
g_{4, b}=\frac{1}{2} \sum_{k=1}^{2}\left|\Re\left(h_{k, 1} h_{k, 2}^{*}\right)\right| .
\end{gathered}
$$

We can see that $g_{4, c}$ given in (23a) is the same as the conventional diversity gain of (13a) when we set $M=4$, and the feedback diversity gain $g_{4, b}$ of (23b) is always non-negative with totally 2 bits of feedback ( 1 bit for each sub-group) as opposed to the $2 \times$ $4-4=4$ bits feedback described for the general case.

Similarly, for systems with $M=3$ antennas, we have $M_{1}=2$ and $M_{2}=1$. Since there is only 1 antenna in sub-group 2, we have $\mathbf{w}_{2}=1 / \sqrt{3}$. For the first subgroup with 2 transmit antennas, we apply the space encoding vector $\mathbf{w}_{1}$ as defined in (20). With this encoding scheme, the receiver SNR can be computed from (9) as

$$
\gamma_{3}=\left(g_{3, c}+g_{3, b}\right) \cdot \gamma_{0},
$$

with the conventional diversity gain $g_{3, c}$ and feedback diversity gain $g_{3, b}$ being given by

$$
\begin{gathered}
g_{3, c}=\frac{1}{3}\left(\sum_{m=1}^{2}\left|h_{1, m}\right|^{2}+\left|h_{2,1}\right|^{2}\right), \\
g_{3, b}=\frac{2}{3}\left|\Re\left(h_{1,1} h_{1,2}^{*}\right)\right| .
\end{gathered}
$$

When there are only two transmit antennas present in the system, we have $w_{1}=w_{2}=1 / \sqrt{2}$, and this scheme reduced to orthogonal space time block code.

With the method presented in this subsection, we only need 1 bit and 2 bits feedback information for systems with $M=3$ and $M=4$ transmit antennas, respectively.

\section{SIMULATION RESULTS}

Simulations are carried out for 3GPP HSDPA systems to evaluate the performance of the proposed adaptive diversity scheme. The simulation parameters are defined according to the HSDPA technical specifications [1] and are shown in Table I. In the simulation, the fading channels are assumed to be 
Table I. Simulation Parameters for 3GPP HSDPA Systems

\begin{tabular}{ll}
\hline Carrier Frequency & $2 \mathrm{GHz}$ \\
Spreading Factor $(\mathrm{SF})$ & 16 \\
Number of Multicodes $\left(N_{c}\right)$ & 10 \\
Frame Length & $2 \mathrm{~m}$ \\
Chip Rate $\left(R_{c}\right)$ & $3.84 \mathrm{Mbps}$ \\
CPICH power & $10 \% I_{o r}$ \\
$E_{c} / I_{o r}$ & $70 \%$ \\
$I_{o r} / I_{o c}$ & variable \\
Channel Coding & Turbo, rate $R_{t}=3 / 4$ \\
Fading Model & one path Rayleigh \\
Correlation Model & i.i.d. \\
Channel Estimation & perfect \\
Modulation & 64 QAM \\
Feedback Delay & 4 TTI \\
\hline
\end{tabular}

identically independent distributed, even though the method works well under correlated fading scenario.

The frame error rate (FER) performance for systems with 3 transmit antennas and 4 transmit antennas is shown in Figures 3 and 4, respectively. For comparison purpose, the performance of orthogonal space time block coding with 2 transmit antennas are also depicted in these two figures. Comparing the performance of the proposed algorithms with optimum quantized TxAA scheme [11] with the same amount of feedback, we can see that for FER $=1 \%$, the performance improvements are approximately $4 \mathrm{~dB}$ for systems with 3 transmit antennas, and $2 \mathrm{~dB}$ for systems with 4 transmit antennas. Moreover, the performance differences between the two space encoding methods of Section 3.1 and 3.2 are within the range of $0.5 \mathrm{~dB}$.

\section{CONCLUSIONS}

An adaptive space time transmit diversity scheme is proposed for next generation wireless communication systems. Combining an orthogonal space time block code with adaptive sub-group antenna encoding, this close loop diversity scheme can be used for systems with more than two transmit antennas without introducing interference among the transmitted signals. A new quadrant phase constraining method is proposed for the computation of the feedback information and the formulation of the space encoding vectors. For general systems with $M$ transmit antennas, the amount of feedback information required by the encoders are $2 M-4$ bits. For the special cases of systems with 3 and 4 transmit antennas, which are of practical interests for emerging wireless communication systems, the size of feedback information can be as few as 1 bit and 2 bits, respectively. Simulation results show that this new diversity scheme outperforms not only open loop diversity techniques, but also some close loop diver-

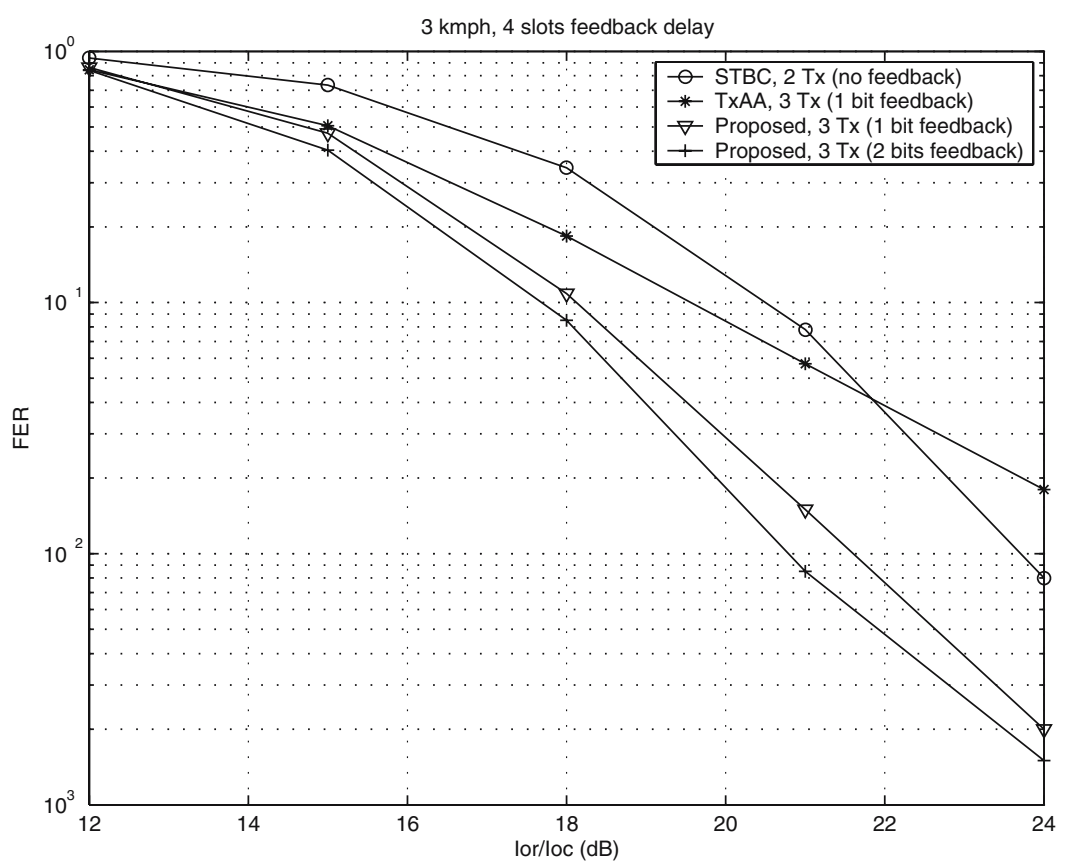

Fig. 3. Comparison of various transmit diversity schemes for systems with 3 transmit antennas. 


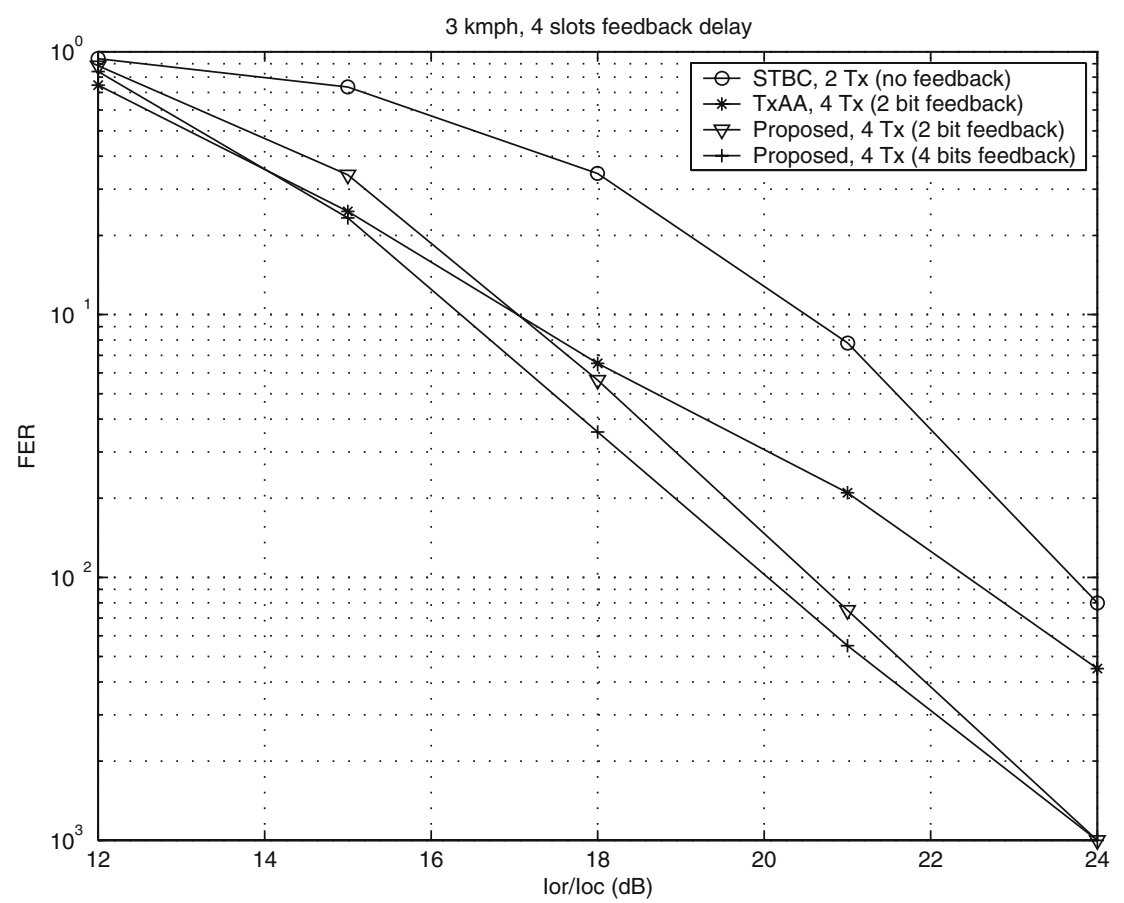

Fig. 4. Comparison of various transmit diversity schemes for systems with 4 transmit antennas.

sity techniques with the same amount of feedback information.

\section{ACKNOWLEDGMENTS}

This work was supported in part by the National Science Foundation under Grant CCF-0514770 and the University of Missouri-Columbia Research Council under Grant URC-05-064.

\section{REFERENCES}

1. 3GPP: 3GPP TR25.848 v4.0.0, "3GPP technical report: Physical layer aspects of ultra high speed downlink packet access,", Mar. 2001. (available at http://www.3gpp.org).

2. S. M. Alamouti, A simple transmit diversity technique for wireless communications, IEEE J. Select. Area Commun., Vol. 16, No. 10, pp. 1451-1458, 1998.

3. V. Tarokh, H. Jafarkhani, and A. R. Calderbank, Space-time block codes from orthogonal designs, IEEE Trans. Info. Theory, Vol. 45, No. 7, pp. 1456-1467, 1999.

4. M. Rupp and C. F. Mecklenbrauker, "On extended Alamouti schemes for space-time coding," Proc. IEEE 5th Int. Symp. Wireless Personal Multimedia Commun., Vol. 1, pp. 115-119, Oct. 2002.

5. A. Hottinen and O. Tirkkonen, "A randomization technique for non-orthogonal space-time block codes," Proc. IEEE Veh. Techno. Conf. VTC'01 Spring, pp. 1479-1482, May 2001.
6. O. Tirkkonen, A. Boariu, and A. Hottinen, "Minimal nonorthogonality rate 1 space-time block code for $3+\mathrm{Tx}$ antennas," Proc. IEEE 6th Int. Symp. Spread-Spectrum Tech., pp. 429-432, Sep. 2000.

7. Siemens:TSGR1-00-0852 "STTD coding method using up to four antennas to improve the performance of indicator channels (PICH)," July, 2000. (available at http://www.3gpp.org).

8. Nokia: TSGR1-01-0829 "Demonstration of a 4-Tx-STTD OL diversity scheme," Aug., 2001. (available at http:// www.3gpp.org).

9. Huawei: TSGR1-03-0016 "Performances of CL-4-Tx-STTD based on ASTTD weighting," Jan., 2003. (available at http:// www.3gpp.org).

10. Y. Xin, Z. Wang, and G. B. Giannakis, Space-time diversity systems based on linear constellation precoding, IEEE Trans. Wireless Commun., Vol. 2, No. 3, pp. 294-309, 2003.

11. R. W. Heath Jr. and A. Paulraj, "A simple scheme for transmit diversity using partial channel feedback," in Proc. IEEE 32nd Asilomar Conf. Signals, Systems and Computers, pp. 1073-1078, 1998.

12. G. Jongren, M. Skoglund, and B. Ottersten, Combining beamforming and orthogonal space-time block coding, IEEE Trans. Info. Theory, Vol. 48, No. 3, pp. 611-627, 2002.

13. S. Zhou and G. B. Giannakis, Optimal transmitter eigenbeamforming and space-time block coding based on channel mean feedback, IEEE Trans. Signal Processing, Vol. 50, No. 10, pp. 2599-2613, 2002.

14. K. Rohani, M. Harrison, and K. Kuchi, "A comparison of base station transmit diversity methods for third generation cellular standards," in Proc. IEEE Veh. Techno. Conf. VTC'99 Spring, pp. 351-355, May, 1999.

15. R. T. Derryberry, S. D. Gray, D. M. Ionescu, G. Mandyan, and B. Raghothaman, Transmit diversity in 3G CDMA systems, IEEE Commun. Mag, Vol. 40, No. 4, pp. 68-75, 2002. 


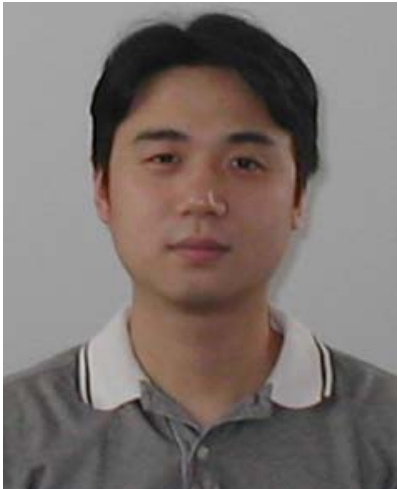

Jingxian Wu received the B.S. (EE) degree from the Beijing University of Aeronautics and Astronautics, Beijing, China, in 1998, the M.S. (EE) degree from Tsinghua University, Beijing, China, in 2001, and the Ph.D. degree from the University of Missouri at Columbia, MO, USA, in 2005. Dr. Wu is an Assistant Professor at the Department of Engineering Science, Sonoma State University, Rohnert Park, CA, USA. His research interests mainly focus on the physical layer of wireless communication systems, including error performance analysis, space-time coding, channel estimation and equalization, and spread spectrum communications. Dr. Wu is a member of Tau Beta Pi.

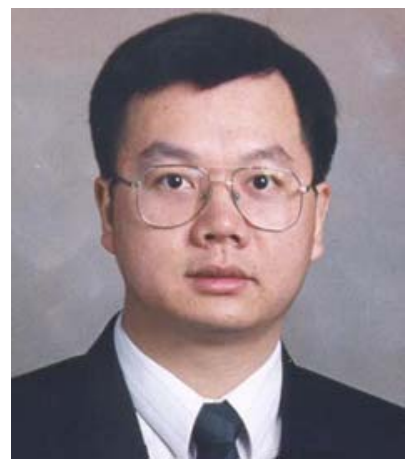

Chengshan Xiao received the B.S. degree from the University of Electronic Science and Technology of China, Chengdu, China, in 1987, the M.S. degree from Tsinghua University, Beijing, China, in 1989, and the Ph.D. degree from the University of Sydney, Sydney, Australia, in 1997, all in electrical engineering. From 1989 to 1993, he was on the Research Staff and then became a Lecturer with the Department of Electronic Engineering at Tsinghua University, Beijing, China. From 1997 to 1999, he was a Senior Member of Scientific Staff at Nortel Networks, Ottawa, ON, Canada. From 1999 to 2000, he was an Assistant Professor with the Department of Electrical and Computer Engineering at the University of Alberta, Edmonton, AB, Canada. Currently, he is a faculty member with the Department of Electrical and Computer Engineering at the University of Missouri, Columbia, Missouri, USA. His research interests include wireless communication networks, signal processing, and multidimensional and multirate systems. He has published extensively in these areas. Dr. Xiao holds three United States patents in wireless communication area. His algorithms have been implemented into Nortel's base station radios with successful technical field trials and network integrations. Dr. Xiao is an Associate Editor for the IEEE Transactions on Wireless Communications. Previously, he was an Associate Editor for the IEEE Transactions on Vehicular Technology, the IEEE Transactions on Circuits and Systems-I, and the international journal of Multidimensional Systems and Signal Processing. $\mathrm{He}$ is also a Co-Chair of the 2005 IEEE Globecom Wireless Communications Symposium. Dr. Xiao is currently serving as the Vice-Chair of the IEEE Communications Society Technical Committee on Personal Communications.

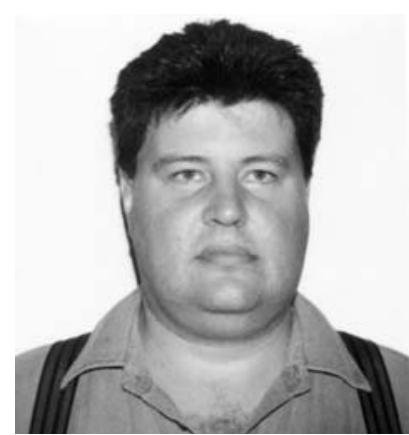

Jan C. Olivier received the B.Eng., M.Eng, and Ph.D. degrees summa cum laude from the University of Pretoria in 1985, 1986, and 1990, respectively. He was with the National Institute for Defense Research in Pretoria, South Africa until 1992, then with Potchefstroom University and University of Pretoria as an Associate Professor until 1995. He was a Member of Scientific Staff at Bell Northern Research (BNR) Ottawa, Canada until 1999, and a Principle Scientist at Nokia Research Laboratory in Texas, U.S.A. until July 2003, when he joined the University of Pretoria as a Professor. He's research interests include Estimation, Detection, Inference and Numerical Methods. 